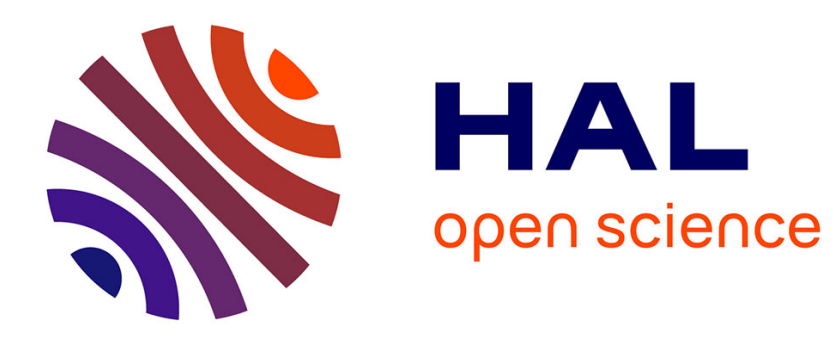

\title{
Stability of immature cell dynamics in healthy and unhealthy hematopoiesis
}

\author{
Walid Djema, Frédéric Mazenc, Catherine Bonnet
}

\section{To cite this version:}

Walid Djema, Frédéric Mazenc, Catherine Bonnet. Stability of immature cell dynamics in healthy and unhealthy hematopoiesis. 2016 American Control Conference (ACC), Jul 2016, Boston, United States. pp.6121 - 6126, 10.1109/ACC.2016.7526631 . hal-01389865v2

\section{HAL Id: hal-01389865 \\ https://hal.inria.fr/hal-01389865v2}

Submitted on 9 Jan 2017

HAL is a multi-disciplinary open access archive for the deposit and dissemination of scientific research documents, whether they are published or not. The documents may come from teaching and research institutions in France or abroad, or from public or private research centers.
L'archive ouverte pluridisciplinaire HAL, est destinée au dépôt et à la diffusion de documents scientifiques de niveau recherche, publiés ou non, émanant des établissements d'enseignement et de recherche français ou étrangers, des laboratoires publics ou privés. 


\title{
Stability of Immature Cell Dynamics in Healthy and Unhealthy Hematopoiesis
}

\author{
Walid Djema, Frédéric Mazenc, Catherine Bonnet
}

\begin{abstract}
A nonlinear system with distributed delays is studied. The model takes into account the fast self-renewal dynamics observed in Acute Myeloid Leukemia (AML). Stability and instability conditions are derived for the zero equilibrium of the AML model. Notice that, biologically, the zero equilibrium means the eradication of all malignant cells, which is the aim of the anti-AML therapy. The novelty of this work is that we consider time-varying biological parameters reflecting the fact that the differentiating and self-renewing parameters become time-variant both under the effect of the disease and the drugs. A simpler case of the studied model is well suited to describe the healthy hematopoiesis. In this case, we focus on the stability of the positive steady state since it reflects the surviving of all healthy blood cells. Via a construction of a novel Lyapunov functional, we derive sufficient conditions for the local exponential stability of the favourable equilibrium and we propose an estimate of its basin of attraction.
\end{abstract}

Key Words: Time-delay Systems, Lyapunov functionals.

\section{INTRODUCTION}

Time-delay systems (TDS) appear in many modelling areas such as traffic flow, chemistry, communication networks, economics and biology (see [5], [9], and references therein). The stability analysis of these models is a key issue and it is well known that the Lyapunov theory makes it possible, in some cases, to estimate rates of convergence of solutions, to establish ISS robustness results and to determine approximations of the basins of attraction for locally asymptotically stable equilibrium points (see [12], [7], [8] for more information). However, the search for a suitable Lyapunov functional for nonlinear TDS is generally quite difficult, since no systematic methods apply [7], and this is the case of the studied model of hematopoiesis.

In a healthy bone marrow, the hematopoeitic stem cells (HSC) develop into white blood cells, red blood cells, platelets and many other blood cells. The HSC are immature cells able to produce cells with the same maturity level and to differentiate into more mature and specific cells. In the case of acute myelogenous leukemia (AML), an overproduction of abnormal blasts is observed. These malignant cells are unable to develop further into mature white blood cells and prevent production of adequate numbers of other types of healthy blood cells. The mathematical model that describes healthy or unhealthy hematopoiesis is given by nonlinear transport

Walid Djema, Frédéric Mazenc and Catherine Bonnet are with Inria, Université Paris-Saclay, L2S-CentraleSupélec, 3 rue Joliot Curie, 91192, Gif-sur-Yvette, France. walid.djema@inria.fr, frederic.mazencdi2s.centralesupelec.fr, catherine.bonneteinria.fr.

This work is supported by a public grant overseen by the French National Research Agency (ANR) through the "iCODE Institute project" funded by the IDEX Paris-Saclay. equations, which are transformed to a nonlinear system with distributed delays using the method of characteristics. The new results in this contribution are outlined according to the following organization of the paper:

In Section II, we complete the analysis of the zero equilibrium of the multi-stage AML model analyzed recently in [2] and [4], by considering the case of time-varying differentiating, self-renewing and fast self-renewing rates.

In Section III, we focus on the model without fast selfrenewal dynamics and with constant biological parameters. The latter case is well suited to describe the process of healthy hematopoiesis. This simpler model was proposed by Adimy in [1] and partially analyzed in [10] and [3]. Recall that the positive equilibrium is the most interesting from a practical point of view because it models the normal process of hematopoiesis, where all the generations of healthy cells survive. Moreover, several diseases may disrupt strongly the hematopoiesis process and a stability analysis of the linear approximation is insufficient. Indeed, as mentioned in [10], estimates of the domain of attraction of the positive equilibrium point and of the exponential decay rate for the nonlinear full-order system have not been found and these are still important open problems. In the present paper, first, exponential stability of the positive steady state is established and decay rate of solutions are approximated. Moreover, an open subset of its basin of attraction is provided via a construction of a novel Lyapunov functional. This region depends on the different biological parameters involved in the biological process of hematopoiesis.

\section{Stability OF THE MODEL WITH FAST SELF-RENEWAL DYNAMICS}

A schematic representation of the cell dynamics is given in Figure 1. For all $i \in I_{n}=\{1, \ldots, n\}$, by $x_{i}$ we denote the total population density of resting cells and by $\tilde{x}_{i}$ the fast self-renewing cells, which have $n \geq 1$ stages of maturity.

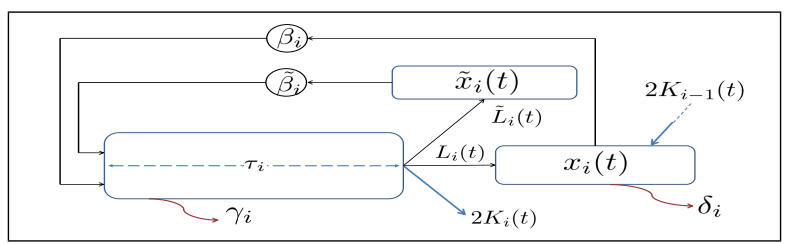

Fig. 1. Schematic representation of the age-structured model describing unhealthy hematopoeisis.

The constant $\delta_{i}$ is the death rate of quiescent cells. The function $g_{i}(a)$ is defined by $g_{i}(a)=e^{-\gamma_{i} a} f_{i}(a)$ where $f_{i}$ is a density function describing the mitosis and is such 
that $\int_{0}^{\tau_{i}} f_{i}(a) d a=1$, and $\gamma_{i}$ is the death rate of the proliferating cells (apoptosis). Proliferating cells can divide between the moment they enter the proliferating phase and a maximal age $\tau_{i}>0$. The reintroduction functions $\beta_{i}\left(x_{i}\right)$ and $\tilde{\beta}_{i}\left(\tilde{x}_{i}\right)$ from the resting to the proliferating phases are considered to be nonlinear, continuous, decreasing functions, and $\lim _{a \rightarrow+\infty} \tilde{\beta}_{i}(a)=\lim _{a \rightarrow+\infty} \beta_{i}(a)=0$.

The total population densities $x_{i}$ and $\tilde{x}_{i}$ are described by the following time-delay system, for all $i \in I_{n}=\{1, \ldots, n\}$,

$$
\left\{\begin{aligned}
\dot{x}_{i}(t)= & -\left(\delta_{i}+\beta_{i}\left(x_{i}(t)\right) x_{i}(t)+L_{i}(t) \Im_{i}\left(w_{i}, \tilde{w}_{i}\right)\right. \\
& +2 K_{i-1}(t) \Im_{i-1}\left(w_{i-1}, \tilde{w}_{i-1}\right) \\
\dot{\tilde{x}}_{i}(t)= & -\tilde{w}_{i}\left(\tilde{x}_{i}(t)\right)+\tilde{L}_{i}(t) \Im_{i}\left(w_{i}, \tilde{w}_{i}\right), \quad t \geq 0,
\end{aligned}\right.
$$

where, with an abuse of notation, $\Im_{i}\left(w_{i}, \tilde{w}_{i}\right)=$ $\int_{0}^{\tau_{i}} g_{i}(a)\left[w_{i}\left(x_{i}(t-a)\right)+\tilde{w}_{i}\left(\tilde{x}_{i}(t-a)\right)\right] d a$, where $a$ is the age of cells. We assume that the functions $L_{i}(\cdot)$ and $\tilde{L}_{i}(\cdot)$ are of class $C^{0}$, for all $i \in I_{n}$, such that $L_{i}(t):=2 \sigma_{i}(t)(1-$ $\left.K_{i}(t)\right), \quad \tilde{L}_{i}(t):=2\left(1-\sigma_{i}(t)\right)\left(1-K_{i}(t)\right), K_{0}=0$, for all $t \geq 0$. The rates $K_{i}(t)$ represent the proportion of cells that differentiate, while the functions $1-\sigma_{i}(t)$ characterize the probability of fast self-renewal at the time instant $t$. Moreover, we introduce for later use the parameters

$$
C_{i}=\int_{0}^{\tau_{i}} g_{i}(a) d a, \text { for all } i \in I_{n} .
$$

Originally, the model described by (1) was introduced in [2] with constant parameters $K_{i}, L_{i}, \tilde{L}_{i}$ and $\sigma_{i}$, for all $i \in I_{n}$. Since AML is characterized by a blockade in the differentiation process, which becomes also a target for some drugs already used in the treatment of this disease, we consider in this paper the time-varying system (1).

Since each proliferating cell, which does not die by apoptosis and completes its mitosis, gives birth to two daughter cells, we deduce that $K_{i}(t) \in[0,1]$ for all $t \geq 0$. In fact, we assume that $K(t) \in\left[K_{i \text { min }}, K_{i \text { max }}\right] \subset(0,1)$, for all $t \geq 0$, meaning that even for an unhealthy hematopoiesis, the differentiating process will not completely vanish. More precisely, in the AML case, due to the blockade in the differentiation process, we expect that $\lim _{t \rightarrow \infty} K(t)=K_{i \min } \neq$ 0 . Similarly, we consider the case where for all $t \geq 0, \sigma_{i}(t) \in$ $\left[\sigma_{i \min }, \sigma_{i \max }\right] \subseteq[0,1]$. From medical practice we know that, in the AML case, a blockade in the differentiation process (i.e. $\left.\lim _{t \rightarrow \infty} K_{i}(t)=K_{i \min }\right)$ and a high rate of fast selfrenewing (i.e. $\lim _{t \rightarrow \infty} \sigma_{i}(t)=\sigma_{i \min }$ ) are simultaneously observed. This fact yields to $\lim _{t \rightarrow \infty} \tilde{L}_{i}(t)=\tilde{L}_{i \max }$, in the AML case, with $\tilde{L}_{i \max }=2\left(1-\sigma_{i \min }\right)\left(1-K_{i \min }\right)$. The situation where $\sigma_{i}=0$ describes the worst leukemic case in which all the proliferating cells, that complete their mitosis, will choose the fast self-renewing process. On the other hand, the case where $\sigma_{i}=1$, and all the parameters involved in the model are constant, is well suited to the healthy hematopoiesis as discussed in Section III.

Moreover, we define for all $i \in I_{n}$,

$$
\hat{L}_{i}(t):=L_{i}(t)+\tilde{L}_{i}(t)=2\left(1-K_{i}(t)\right), \quad t \geq 0 .
$$

Notice that $\hat{L}(t) \in\left[\hat{L}_{i \min }, \hat{L}_{i \max }\right] \subset(0,2)$, for all $t>0$, where $\hat{L}_{i \max }=2\left(1-K_{i \min }\right)$.
It is easy to prove that system (1) is a positive system (i.e. for nonnegative initial conditions, the solutions are nonnegative). Throughout this section, we employ the positivity of the system to construct a suitable Lyapunov-Krasovskii functional in order to investigate the stability properties of the overall system. We start with the following Proposition:

Proposition 1: If the condition

$$
C_{i} \tilde{L}_{i \max }<1
$$

for all $i \in I_{n}$, is not satisfied, then there exists a system in the family of systems (1) whose origin is not globally asymptotically stable.

Proof: We prove Proposition 1 by contradiction. Let us assume that (3) is not satisfied for a given $j \in I_{n}$ and that all the positive solutions of the system (1), with for all $i \in I_{n}$, $\tilde{L}_{i}(t)=\tilde{L}_{i \max }$, for all $t \geq 0$, converges to the origin.

Observe that

$$
\dot{\tilde{x}}_{i}(t) \geq-\tilde{w}_{i}\left(\tilde{x}_{i}(t)\right)+\tilde{L}_{i \max } \int_{t-\tau_{i}}^{t} g_{i}(t-a) \tilde{w}_{i}\left(\tilde{x}_{i}(a)\right) d a
$$

Let us introduce the functionals

$$
h_{i}\left(\tilde{x}_{i t}\right)=\int_{t-\tau_{i}}^{t} \int_{m}^{t} g_{i}\left(m-a+\tau_{i}\right) \tilde{w}_{i}\left(\tilde{x}_{i}(a)\right) d a d m .
$$

Simple calculations give

$$
\dot{h}_{i}(t)=C_{i} \tilde{w}_{i}\left(\tilde{x}_{i}(t)\right)-\int_{t-\tau_{i}}^{t} g_{i}(t-a) \tilde{w}_{i}\left(\tilde{x}_{i}(a)\right) d a .
$$

From (4) and (6), it follows that the derivatives of the functionals $H_{i}\left(\tilde{x}_{i t}\right)=\tilde{x}_{i}(t)+\tilde{L}_{i \max } h_{i}\left(\tilde{x}_{i t}\right)$, along the trajectories of (1), satisfy

$$
\dot{H}_{i}(t) \geq\left(C_{i} \tilde{L}_{i \max }-1\right) \tilde{w}_{i}\left(\tilde{x}_{i}(t)\right) .
$$

Since there exists $j \in I_{n}$ such that $C_{j} \tilde{L}_{j \max }>1$, we get $\dot{H}_{j}(t) \geq 0$. It follows that, $H_{j}\left(\tilde{x}_{j t}\right) \geq H_{j}(0)>0$. Therefore, $H_{j}\left(\tilde{x}_{j t}\right)$ does not converge to zero. But we consider a solution that converges to the origin, which implies that $H_{j}\left(\tilde{x}_{j t}\right)$ converges to zero. This yields a contradiction.

Remark 1: If $\sigma_{i \min }=0$, we obtain $\hat{L}_{i \max }=\tilde{L}_{i \max }$. Therefore, from Proposition 1, we observe that if the condition $C_{i} \hat{L}_{i \max }<1$ is not satisfied for all $i \in I_{n}$, then there exists a system (1) such that its origin is not globally asymptotically stable. Consequently, we can not establish global exponential stability of the origin of system (1) if the condition $C_{i} \hat{L}_{i \max }>1$ holds for some $i \in I_{n}$.

Now, let us state and prove the following result:

Theorem 1: For all $i \in I_{n}$, if the conditions

$$
C_{i} \hat{L}_{i \max }<1
$$

are satisfied, then all the positive trajectories of (1) converge exponentially to the origin.

Proof: First, we observe that when the conditions (8) are satisfied, then for all $\lambda_{i} \in\left(1, \frac{1+C_{i} \hat{L}_{i \max }}{2 C_{i} \hat{L}_{i \max }}\right)$, the following inequalities are verified

$$
1-\lambda_{i} C_{i} \hat{L}_{i \max }>\frac{1-C_{i} \hat{L}_{i \max }}{2}>0 .
$$


Next, let us introduce, for all $i \in I_{n}$, the functionals defined along the trajectories of system (1), by

$$
\begin{aligned}
v_{i}\left(x_{i t}, \tilde{x}_{i t}\right)=\int_{t-\tau_{i}}^{t} & \int_{m}^{t} e^{-\rho_{i}\left(t-m-\tau_{i}\right)} g_{i}\left(m-a+\tau_{i}\right) \\
& \times\left[w_{i}\left(x_{i}(a)\right)+\tilde{w}_{i}\left(\tilde{x}_{i}(a)\right)\right] d a d m
\end{aligned}
$$

where $\rho_{i}$ are strictly positive constants to be selected later. The derivatives of the functionals (10), along the trajectories of (1), satisfy

$$
\begin{aligned}
\dot{v}_{i}(t) \leq & -\rho_{i} v_{i}\left(x_{i t}, \tilde{x}_{i t}\right)-\Im_{i}\left(w_{i}, \tilde{w}_{i}\right) \\
& +\left[w_{i}\left(x_{i}(t)\right)+\tilde{w}_{i}\left(\tilde{x}_{i}(t)\right)\right] e^{\rho_{i} \tau_{i}} C_{i} .
\end{aligned}
$$

Let us introduce the following functional for the first compartment of unhealthy cells

$$
\mathcal{V}_{1}\left(x_{1 t}, \tilde{x}_{1 t}\right)=x_{1}(t)+\tilde{x}_{1}(t)+\lambda_{1} \hat{L}_{1 \max } v_{1}\left(x_{1 t}, \tilde{x}_{1 t}\right),
$$

where $\lambda_{1}$ satisfies (9). Using (11), it follows that

$$
\begin{aligned}
\dot{\mathcal{V}}_{1}(t) \leq & -\left[\delta_{1}+\left(1-\lambda_{1} e^{\rho_{1} \tau_{1}} \hat{L}_{1 \max } C_{1}\right) \beta_{1}\left(x_{1}(t)\right)\right] x_{1}(t) \\
& -\left(1-\lambda_{1} e^{\rho_{1} \tau_{1}} \hat{L}_{1 \max } C_{1}\right) \tilde{w}_{1}\left(\tilde{x}_{1}(t)\right) \\
& -\lambda_{1} \rho_{1} \hat{L}_{1 \max } v_{1}\left(x_{1 t}, \tilde{x}_{1 t}\right) \\
& +\left(\hat{L}_{1}(t)-\lambda_{1} \hat{L}_{1 \max }\right) \Im_{1}\left(w_{1}, \tilde{w}_{1}\right) .
\end{aligned}
$$

Since $\lambda_{1}$ satisfies (9). It follows that by selecting any

$$
\rho_{1} \in\left(0, \frac{1}{\tau_{1}} \ln \left(\frac{1+\lambda_{1} C_{1} \hat{L}_{1 \max }}{2 \lambda_{1} C_{1} \hat{L}_{1 \max }}\right)\right),
$$

we obtain $1-\lambda_{1} e^{\rho_{1} \tau_{1}} \hat{L}_{1 \max } C_{1}>\frac{1-\lambda_{1} \hat{L}_{1 \max } C_{1}}{2}>0$. Moreover, since $\hat{L}_{1}(t) \leq \hat{L}_{1 \max }$, we deduce that (13) gives

$$
\begin{aligned}
\dot{\mathcal{V}}_{1}(t) \leq & -\left[\delta_{1}+\theta_{1} \beta_{1}(0)\right] x_{1}(t)-\theta_{1} \tilde{w}\left(\tilde{x}_{1}(t)\right) \\
& -\lambda_{1} \hat{L}_{1 \max } \rho_{1} v_{1}\left(x_{1 t}, \tilde{x}_{1 t}\right)-\bar{\lambda}_{1} \Im_{1}\left(w_{1}, \tilde{w}_{1}\right),
\end{aligned}
$$

where the constants $\theta_{1}=1-\lambda_{1} e^{\rho_{1} \tau_{1}} \hat{L}_{1 \max } C_{1}$ and $\bar{\lambda}_{1}=$ $\left(\lambda_{1}-1\right) \hat{L}_{1 \max }$ are strictly positive. Since the right hand side of (15) is always nonpositive, we deduce that by integrating (15), we obtain for all $t \geq 0$,

$$
\mathcal{V}_{1}\left(x_{1 t}, \tilde{x}_{1 t}\right) \leq \mathcal{V}_{1}\left(\varphi_{x_{1}}, \varphi_{\tilde{x}_{1}}\right)
$$

This means in particular that $\tilde{x}(t)$ is bounded by a constant $\tilde{x}_{1 b}>0$. Since the function $\tilde{\beta}_{1}$ is decreasing, it follows that, for all $t \geq 0$, the inequality (15) gives

$$
\begin{aligned}
\dot{\mathcal{V}}_{1}(t) \leq & -\left[\delta_{1}+\theta_{1} \beta_{1}(0)\right] x_{1}(t)-\theta_{1} \tilde{\beta}_{1}\left(\tilde{x}_{1 b}\right) \tilde{x}_{1}(t) \\
& -\lambda_{1} \hat{L}_{1 \max } \rho_{1} v_{1}\left(x_{1 t}, \tilde{x}_{1 t}\right)-\bar{\lambda}_{1} \Im_{1}\left(w_{1}, \tilde{w}_{1}\right) .
\end{aligned}
$$

We conclude that for all $t \geq 0$,

$$
\dot{\mathcal{V}}_{1}(t) \leq-\tilde{s}_{1} \mathcal{V}_{1}\left(x_{1 t}, \tilde{x}_{1 t}\right)-\bar{\lambda}_{1} \Im_{1}\left(w_{1}, \tilde{w}_{1}\right),
$$

where, $\tilde{s}_{1}=\min \left\{\delta_{1}+\theta_{1} \beta_{1}(0), \theta_{1} \tilde{\beta}_{1}\left(\tilde{x}_{1 b}\right), \lambda_{1} \rho_{1} \hat{L}_{1 \max }\right\}$.

By virtue of the functional $\mathcal{V}_{1}$, we conclude that the origin of the subsystem $i=1$ is globally exponentially stable, with a decay rate smaller than $\tilde{s}_{1}$. Next, in the rest of the proof, we consider the case where the conditions (8) are satisfied for any number of compartments $\left(i \in I_{n}\right)$. Let us introduce the following functional that takes into account the first and the second generations of cells:

$$
\begin{aligned}
\mathcal{V}_{2}\left(X_{2 t}\right)= & x_{2}(t)+\tilde{x}_{2}(t)+\lambda_{2} \hat{L}_{2 \max } v_{2}\left(x_{2 t}, \tilde{x}_{2 t}\right) \\
& +\frac{2 K_{1 \max }}{\bar{\lambda}_{1}} \mathcal{V}_{1}\left(x_{1 t}, \tilde{x}_{1 t}\right)
\end{aligned}
$$

with $X_{2}=\left(x_{1}, \tilde{x}_{1}, x_{2}, \tilde{x}_{2}\right)$. Similarly to the case $i=1$, we select $\rho_{2}$ such that the derivative of the functional (19) along the trajectories of (1) satisfies

$$
\begin{aligned}
\dot{\mathcal{V}}_{2}(t) & \leq-\left[\delta_{2}+\theta_{2} \beta_{2}(0)\right] x_{2}(t)-\theta_{2} \tilde{w}_{2}\left(\tilde{x}_{2}(t)\right) \\
& -\lambda_{2} \hat{L}_{2 \max } \rho_{2} v_{2}\left(x_{2 t}, \tilde{x}_{2 t}\right)+2 K_{1}(t) \Im_{1}\left(w_{1}, \tilde{w}_{1}\right) \\
& -\bar{\lambda}_{2} \Im_{2}\left(w_{2}, \tilde{w}_{2}\right)+\frac{2 K_{1 \max }}{\bar{\lambda}_{1}} \dot{\mathcal{V}}_{1}(t)
\end{aligned}
$$

Combining (20) and (18), we deduce that for all $t \geq 0$,

$$
\begin{aligned}
\dot{\mathcal{V}}_{2}(t) \leq & -\left[\delta_{2}+\theta_{2} \beta_{2}(0)\right] x_{2}(t)-\theta_{2} \tilde{\beta}_{2}\left(\tilde{x}_{2 b}\right) \tilde{x}_{2}(t) \\
& -\lambda_{2} \hat{L}_{2 \max } \rho_{2} v_{2}\left(x_{2 t}, \tilde{x}_{2 t}\right)-\bar{\lambda}_{2} \Im_{2}\left(w_{2}, \tilde{w}_{2}\right) \\
& -\frac{2 \tilde{s}_{1} K_{1 \max }}{\bar{\lambda}_{1}} \mathcal{V}_{1}\left(x_{1 t}, \tilde{x}_{1 t}\right) \\
& -2\left(K_{1 \max }-K_{1}(t)\right) \Im_{1}\left(w_{1}, \tilde{w}_{1}\right) .
\end{aligned}
$$

Since $K_{1}(t) \leq K_{1 \max }$, we straightforwardly deduce that there exists $\tilde{s}_{2}>0$ such that, for all $t \geq 0$,

$$
\dot{\mathcal{V}}_{2}(t) \leq-\tilde{s}_{2} \mathcal{V}_{2}\left(X_{2 t}\right)-\lambda_{2} \Im_{2}\left(w_{2}, \tilde{w}_{2}\right)
$$

Next, by induction, one can readily determine constants $\mathfrak{a}_{i}>0, \mathfrak{b}_{i}>0$ and $\tilde{s}_{n}>0$, such that the derivative of the functional

$$
\mathcal{V}_{n}\left(X_{n t}\right)=\sum_{i=1}^{n}\left[x_{i}(t)+\mathfrak{a}_{i} \tilde{x}_{i}(t)+\mathfrak{b}_{i} v_{i}\left(x_{i t}, \tilde{x}_{i t}\right)\right],
$$

with $X_{n}=\left(x_{1}, \tilde{x}_{1}, \ldots, x_{n}, \tilde{x}_{n}\right)$, along the trajectories of (1), satisfies

$$
\dot{\mathcal{V}}_{n}(t) \leq-\tilde{s}_{n} \mathcal{V}_{n}\left(X_{n t}\right), \text { with } \tilde{s}_{n}>0
$$

From the inequality (24) and the properties of the functional $\mathcal{V}_{n}$, we conclude that the origin of the system (1) is globally exponentially stable with a decay rate smaller than $\tilde{s}_{n}$.

\section{Stability OF THE MODEL DESCRIBING HEALTHY HEMATOPOIESIS}

In this section we focus on a simpler form of system (1) which fits well with the healthy hematopoiesis. The model of interest is in the form

$$
\begin{aligned}
\dot{x}_{i}(t)= & -\left(\delta_{i}+\beta_{i}\left(x_{i}(t)\right)\right) x_{i}(t)+2 L_{i} \Im_{i}\left(w_{i}\right) \\
& +2 K_{i-1} \Im_{i-1}\left(w_{i-1}\right),
\end{aligned}
$$

with $\Im_{i}\left(w_{i}\right)=\int_{t-\tau_{i}}^{t} g_{i}(t-a) w_{i}\left(x_{i}(a)\right) d a$, to ease the notation of the distributed delayed terms. Observe that the system (1) is equivalent to the system (25) when $\tilde{x}_{i} \equiv 0$ and $\sigma_{i} \equiv 1$, for all $i \in I_{n}$, as illustrated in Figure 2. In other terms, we consider that the fast self-renewal process is observed only in leukemic cell dynamics (System (1)), but 
not in the healthy hematopoiesis (System (25)). Compared to Section II, the analysis of system (25) is different since the survival of all the healthy cells is the purpose of normal hematopoiesis. More precisely, we focus on the strictly positive steady state $X^{e}=\left(x_{1}^{e}, \ldots, x_{n}^{e}\right), x_{i}^{e}>0$, of system (25), since it reflects the surviving of all the generations of blood cells.

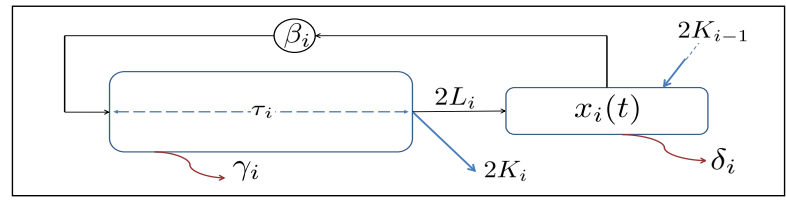

Fig. 2. Schematic representation of the model of healthy hematopoeisis.

All the biological parameters involved in (25) are strictly positive constants with the same definitions introduced in Section II. The rates of differentiation, $K_{i}$, and selfrenewing, $L_{i}$, are positive constants such that $K_{i} \in(0,1)$ and $L_{i}=1-K_{i}$, for all $i \in I_{n}$. Furthermore, we introduce for later use the following parameters, for all $i \in I_{n}$,

$$
\alpha_{i}=2 L_{i} C_{i}-1
$$

where $C_{i}$ are the constants defined in (2). The model described by (25) was first proposed in [1], widely analyzed by an input-output approach in [10] and recently by a Lyapunov approach in [3]. Using a new Lyapunov approach, we extend the results by establishing the local exponential stability under the same conditions that are sufficient for the local asymptotic stability, proposed in [3], and by determining a subset of the basin of attraction of the strictly positive equilibrium of the nonlinear system.

Now, let us recall some useful results:

i) The solutions of system (25) associated with positive initial conditions are positive. Throughout our work, we consider only positive solutions of (25).

ii) We assume that $\alpha_{i}>0$ is satisfied for $i \in I_{n}$. A unique strictly positive equilibrium $X^{e}$ may exist and the following proposition gives a necessary and sufficient condition that ensures its existence.

Proposition 2: Under the previous assumptions, the system (25) admits a strictly positive equilibrium $X^{e}=$ $\left(x_{1}^{e}, \ldots, x_{n}^{e}\right)$ if and only if

$$
\beta_{1}(0)>\delta_{1} / \alpha_{1}
$$

The reader is invited to refer to [3], for the proofs of results given above. In the remainder of this work, we assume that $\alpha_{i}>0$ and that a unique positive steady state $X^{e}$ exists.

We begin the analysis by performing the change of coordinates $\hat{x}_{i}=x_{i}-x_{i}^{e}$. From (25), it follows that

$$
\begin{aligned}
\dot{\hat{x}}_{i}(t)= & -\delta_{i}\left[\hat{x}_{i}(t)+x_{i}^{e}\right]-w_{i}\left(\hat{x}_{i}(t)+x_{i}^{e}\right) \\
& +2 L_{i} \Im_{i}\left(w_{i}\right)+2 K_{i-1} \Im_{i-1}\left(w_{i-1}\right),
\end{aligned}
$$

with $\Im_{i}\left(w_{i}\right)=\int_{t-\tau_{i}}^{t} g_{i}(t-a) w_{i}\left(\hat{x}_{i}(a)+x_{i}^{e}\right) d a$. We are looking for a new representation of (28) that eases the analysis of its origin. Observe that,

$$
w_{i}\left(\hat{x}_{i}+x_{i}^{e}\right)=w_{i}\left(x_{i}^{e}\right)+\mu_{i} \hat{x}_{i}+R_{i}\left(\hat{x}_{i}\right)
$$

where $\mu_{i}=\left[\frac{\partial w_{i}}{\partial x_{i}}\right]_{x_{i}=x_{i}^{e}}=\beta_{i}\left(x_{i}^{e}\right)+\beta_{i}^{\prime}\left(x_{i}^{e}\right) x_{i}^{e}$, and

$$
R_{i}\left(\hat{x}_{i}\right)=\int_{x_{i}^{e}}^{x_{i}^{e}+\hat{x}_{i}}\left[\hat{x}_{i}+x_{i}^{e}-l\right] w_{i}^{(2)}(l) d l .
$$

As an immediate consequence,

$$
\begin{aligned}
\dot{\hat{x}}_{i}(t) & =-\beta_{i *} \hat{x}_{i}(t)+2 L_{i} \mu_{i} \int_{t-\tau_{i}}^{t} g_{i}(t-a) \hat{x}_{i}(a) d a \\
& -R_{i}\left(\hat{x}_{i}(t)\right)+2 L_{i} \int_{t-\tau_{i}}^{t} g_{i}(t-a) R_{i}\left(\hat{x}_{i}(a)\right) d a \\
& +2 K_{i-1} \mu_{i-1} \int_{t-\tau_{i-1}}^{t} g_{i-1}(t-a) \hat{x}_{i-1}(a) d a \\
& +2 K_{i-1} \int_{t-\tau_{i-1}}^{t} g_{i-1}(t-a) R_{i-1}\left(\hat{x}_{i-1}(a)\right) d a
\end{aligned}
$$

with $\beta_{i *}=\delta_{i}+\mu_{i}$. If the solutions of system (31) converge to the origin, then the positive solutions of system (25) converge to the positive steady state $X^{e}$. Due to the shifting, we observe that the solutions of system (31) are not positive. Consequently, contrary to Section II, we can not take advantage from linear functionals constructions. This observation motivates the introduction of the following quadratic function and functionals:

$$
\begin{gathered}
Q(a)=\frac{1}{2} a^{2} \\
\Omega_{i}\left(\varphi_{i t}\right)=\int_{t-\tau_{i}}^{t} \int_{l}^{t} g_{i}\left(l-a+\tau_{i}\right) Q\left(\varphi_{i}(a)\right) d a d l \\
\Lambda_{i}\left(\varphi_{i t}\right)=\int_{t-\tau_{i}}^{t} e^{l-t} \int_{l}^{t} g_{i}\left(l-a+\tau_{i}\right) Q\left(\varphi_{i}(a)\right) d a d l
\end{gathered}
$$

and the following constants

$$
\varsigma_{i}=\beta_{i *}-2 L_{i}\left|\mu_{i}\right| C_{i}=\delta_{i}+\mu_{i}-2 L_{i}\left|\mu_{i}\right| C_{i} .
$$

We observe the similarity between the functionals $\Omega_{i}$ and $\Lambda_{i}$, inspired from the construction of Kolmanovskii and Myshkis [5]. For technical reasons, we use a weighted combination between them to compensate the distributed delayed terms and estimate the exponential decay rate.

\section{A. Exponential stability of the linear approximation}

We extend the results in [3] by designing a novel Lyapunov-Krasovskii functional implying local exponential stability. Let us prove the following result:

Theorem 2: The conditions

$$
\varsigma_{i}>0
$$

for all $i \in I_{n}$, ensure that the origin of (31) is locally exponentially stable.

Proof: The linear approximation of the system (31) at its origin is given by

$$
\begin{aligned}
\dot{z}_{i}(t)= & -\beta_{i *} z_{i}(t)+2 L_{i} \mu_{i} \int_{t-\tau_{i}}^{t} g_{i}(t-a) z_{i}(a) d a \\
& +2 K_{i-1} \mu_{i-1} \int_{t-\tau_{i-1}}^{t} g_{i-1}(t-a) z_{i-1}(a) d a .
\end{aligned}
$$


Let us define for all $i \in I_{n}$ the following functionals

$$
S_{i}\left(z_{i t}\right)=\frac{1}{2} Q\left(z_{i}(t)\right)+L_{i}\left|\mu_{i}\right| \Omega_{i}\left(z_{i t}\right),
$$

and

$$
N_{1}\left(z_{1 t}\right)=S_{1}\left(z_{1 t}\right)+\frac{\varsigma_{1}}{2 C_{1}} \Lambda_{1}\left(z_{1 t}\right),
$$

and for all $i \in\{2, \ldots, n\}$,

$$
N_{i}\left(z_{i t}, z_{i-1 t}\right)=S_{i}\left(z_{i t}\right)+\frac{\varsigma_{i}}{2 C_{i}} \Lambda_{i}\left(z_{i t}\right)+\psi_{i} \Lambda_{i-1}\left(z_{i-1 t}\right),
$$

where $\psi_{i}$ is an appropriate strictly positive constant to be determined later for all $i \in\{2, \ldots, n\}$. First, observe for later use that the derivatives of the functionals $\Omega_{i}$ and $\Lambda_{i}$, for all $i \in I_{n}$, along the trajectories of (37) satisfy,

$$
\begin{aligned}
\dot{\Omega}_{i}(t)= & C_{i} Q\left(z_{i}(t)\right)-\int_{t-\tau_{i}}^{t} g_{i}(t-a) Q\left(z_{i}(a)\right) d a, \\
\dot{\Lambda}_{i}(t) \leq & -\Lambda_{i}\left(z_{i t}\right)-e^{-\tau_{i}} \int_{t-\tau_{i}}^{t} g_{i}(t-a) Q\left(z_{i}(a)\right) d a \\
& +C_{i} Q\left(z_{i}(t)\right),
\end{aligned}
$$

where the last inequality is a consequence of the definition of the constants $C_{i}$ given by (2). Next, For the sake of clarity, we decompose the proof of Theorem 2 in two steps: we start by the first generation of cells $(i=1)$, then we generalize to any number of compartments $i \in I_{n}$.

1) Lyapunov-Krasovskii functional for the first compartment: Using the inequality $\left|z_{1}(t) z_{1}(a)\right| \leq \frac{1}{2}\left|z_{1}(t)\right|^{2}+$ $\frac{1}{2}\left|z_{1}(a)\right|^{2}$, we prove that the derivative along the trajectories of (37) of $Q\left(z_{1}(t)\right)$, where $Q$ is the function defined in (32), satisfies the inequality

$$
\begin{aligned}
\frac{1}{2} \dot{Q}(t) \leq & {\left[-\beta_{1 *}+L_{1}\left|\mu_{1}\right| C_{1}\right] Q\left(z_{1}(t)\right) } \\
& +L_{1}\left|\mu_{1}\right| \int_{t-\tau_{1}}^{t} g_{1}(t-a) Q\left(z_{1}(a)\right) d a .
\end{aligned}
$$

Using (41), (42) and (43), we prove that the derivative of the functional $N_{1}$, defined in (39), along the trajectories of (37), satisfies

$$
\dot{N}_{1}(t) \leq-\frac{\varsigma_{1}}{2} Q\left(z_{1}(t)\right)-\frac{\varsigma_{1}}{2 C_{1}} \Lambda_{1}\left(z_{1 t}\right) .
$$

On the other hand, from the definition of $N_{1}$, we readily check that

$$
N_{1}\left(z_{1 t}\right) \leq \frac{1}{2} Q\left(z_{1}(t)\right)+s_{1} \Lambda_{1}\left(z_{1 t}\right)
$$

with $s_{1}=\frac{\varsigma_{1}+2 L_{1} C_{1}\left|\mu_{1}\right| e^{\tau_{1}}}{2 C_{1}}$. From (44) and (45), we get

$$
\dot{N}_{1}(t) \leq-\tilde{\varsigma}_{1} N_{1}\left(z_{1 t}\right)-\frac{\varsigma_{1}}{4} Q\left(z_{1}(t)\right),
$$

for all $\tilde{\varsigma}_{1} \in\left(0, \min \left\{\frac{\varsigma_{1}}{2}, \frac{\varsigma_{1}}{\varsigma_{1}+2 L_{1} C_{1}\left|\mu_{1}\right| e^{\tau_{1}}}\right\}\right)$.

We conclude from (46) that the origin of the subsystem $(i=1)$ is exponentially stable.

2) Lyapunov-Krasovskii functional for the overall system: Now, using the inequalities: $\left|z_{i}(t) z_{i}(a)\right| \leq Q\left(z_{i}(t)\right)+$ $Q\left(z_{i}(a)\right.$, for all $i \in I_{n}$, and $\left|z_{i}(t) z_{i-1}(a)\right| \leq \xi_{i} Q\left(z_{i}(t)\right)+$ $\frac{1}{\xi_{i}} Q\left(z_{i-1}(a)\right)$, with $\xi_{i}>0$, for all $i>1$, we prove that the derivatives of the functions $Q\left(z_{i}(t)\right)$, for all $i>1$, along the trajectories of (37), satisfy

$$
\begin{aligned}
\frac{1}{2} \dot{Q}(t) & \leq\left[-\beta_{i *}+L_{i}\left|\mu_{i}\right| C_{i}\right] Q\left(z_{i}(t)\right) \\
& +L_{i}\left|\mu_{i}\right| \int_{t-\tau_{i}}^{t} g_{i}(t-a) Q\left(z_{i}(a)\right) d a \\
& +\xi_{i} K_{i-1}\left|\mu_{i-1}\right| C_{i-1} Q\left(z_{i}(t)\right) \\
& +\frac{K_{i-1}\left|\mu_{i-1}\right|}{\xi_{i}} \int_{t-\tau_{i-1}}^{t} g_{i-1}(t-a) Q\left(z_{i-1}(a)\right) d a .
\end{aligned}
$$

We observe that, for $i>1$, the derivatives of the functionals $N_{i}$, defined in (40), along the trajectories of (37), satisfy

$$
\begin{aligned}
& \dot{N}_{i}(t) \leq\left[-\beta_{i *}+2 L_{i}\left|\mu_{i}\right| C_{i}\right] Q\left(z_{i}(t)\right) \\
& +\xi_{i} K_{i-1}\left|\mu_{i-1}\right| C_{i-1} Q\left(z_{i}(t)\right) \\
& +\frac{K_{i-1}\left|\mu_{i-1}\right|}{\xi_{i}} \int_{t-\tau_{i-1}}^{t} g_{i-1}(t-a) Q\left(z_{i-1}(a)\right) d a \\
& -\frac{\varsigma_{i}}{2 C_{i}} \Lambda_{i}\left(z_{i t}\right)+\frac{\varsigma_{i}}{2} Q\left(z_{i}(t)\right)+\psi_{i} \dot{\Lambda}_{i-1}(t) .
\end{aligned}
$$

Let us select, for all $i \in\{2, \ldots, n\}$,

$$
\psi_{i}=\frac{K_{i-1}\left|\mu_{i-1}\right| e^{\tau_{i-1}}}{\xi_{i}} \text {, and } \xi_{i}=\frac{\varsigma_{i}}{4 K_{i-1}\left|\mu_{i-1}\right| C_{i-1}} .
$$

Then, the inequality (47) gives

$$
\begin{aligned}
\dot{N}_{i}(t) \leq & -\frac{\varsigma_{i}}{4} Q\left(z_{i}(t)\right)-\frac{\varsigma_{i}}{2 C_{i}} \Lambda_{i}\left(z_{i t}\right) \\
& -\psi_{i} \Lambda_{i-1}\left(z_{i-1 t}\right)+\psi_{i} C_{i-1} Q\left(z_{i-1}(t)\right) .
\end{aligned}
$$

Similarly to the case $i=1$, (44)-(46), we easily determine a strictly positive real number $\tilde{\varsigma}_{i}$ such that

$$
\begin{aligned}
\dot{N}_{i}(t) \leq & -\tilde{\varsigma}_{i} N_{i}\left(z_{i t}, z_{i-1 t}\right)-\frac{\varsigma_{i}}{8} Q\left(z_{i}(t)\right) \\
& +\frac{\theta_{i} \varsigma_{i-1}}{8} Q\left(z_{i-1}(t)\right),
\end{aligned}
$$

with $\theta_{i}=\frac{8 \psi_{i} C_{i-1}}{\varsigma_{i-1}}$. Finally, we introduce the functional:

$$
W\left(Z_{t}\right)=p_{1} N_{1}\left(z_{1 t}\right)+\sum_{i=2}^{n} p_{i} N_{i}\left(z_{i t}, z_{i-1 t}\right),
$$

with $p_{i}=2^{n-i} \prod_{k=i+1}^{n} \theta_{k}$, and $p_{n}=1$. From (46) and (50), we conclude that

$$
\begin{aligned}
\dot{W}(t) & \leq-p_{1} \tilde{\varsigma}_{1} N_{1}\left(z_{1 t}\right)-\sum_{i=2}^{n} p_{i} \tilde{\varsigma}_{i} N_{i}\left(z_{i t}, z_{i-1 t}\right) \\
& -\sum_{i=1}^{n} \frac{p_{i} \varsigma_{i}}{8} Q\left(z_{i}(t)\right)+\sum_{i=2}^{n} \frac{p_{i} \theta_{i} \varsigma_{i-1}}{8} Q\left(z_{i-1}(t)\right) .
\end{aligned}
$$

Noticing that $p_{i} \theta_{i}=\frac{1}{2} p_{i-1}$, we easily determine a strictly positive constant $\tilde{\varsigma}$ such that

$$
\dot{W}(t) \leq-\tilde{\varsigma} W\left(Z_{t}\right),
$$

To summarize, by the virtue of the functionals $N_{i}$, for all $i \in I_{n}$, and the inequality (53) we conclude that the origin of the system (37) is exponentially stable. 


\section{B. Exponential stability of the nonlinear system}

The next step consists in determining an approximation of the basin of attraction of the strictly positive equilibrium of the nonlinear system (31). To achieve this, we consider the case where

$$
\beta_{i}\left(x_{i}\right)=\frac{\beta_{i}(0)}{1+b_{i} x_{i}^{n_{i}}},
$$

with $\beta_{i}(0)>0, b_{i}>0$ and $n_{i}>2$. This typical choice of the functions $\beta_{i}$ was justified by Mackey in [6], then assumed in [11], [1] and [10]. After long but simple calculations, using the expression of $\beta_{i}$ in (54), we prove that there exist constants $\hat{s}_{i}>0$, for all $i \in I_{n}$, such that

$$
R_{i}\left(\hat{x}_{i}\right) \leq \hat{s}_{i} Q\left(\hat{x}_{i}\right)
$$

where $R_{i}$ are the functions in (30). Let us consider the functionals $S_{i}$ and $N_{i}$ defined in (38), (39) and (40). In order to ease the notation, we denote

$$
I_{i}\left(\hat{x}_{i t}\right)=\int_{t-\tau_{i}}^{t} g_{i}(t-a) Q\left(\hat{x}_{i}(a)\right) d a .
$$

Finally, we define the constants

$$
\tilde{k}_{i}=\frac{\varsigma_{i}}{8 \hat{s}_{i}}, \hat{k}_{i}=\frac{\varsigma_{i}}{4 C_{i} L_{i} \hat{s}_{i} e^{\tau_{i}}} \text { and } \bar{N}_{i}=\min \left\{\tilde{k}_{i}^{2}, \hat{k}_{i}^{2}\right\} \text {. }
$$

Now we are ready to state and prove the following theorem:

Theorem 3: Let the system (31) be such that

$$
\varsigma_{i}>0
$$

for all $i \in I_{n}$. Therefore all the solutions of (31) with initial conditions $\hat{\varphi}_{i} \in \mathcal{C}\left(\left[-\tau_{i}, 0\right], \mathbb{R}\right)$ satisfying

$$
N_{i}\left(\hat{\varphi}_{i}, \hat{\varphi}_{i-1}\right)<\bar{N}_{i}
$$

converge exponentially to the origin.

Notice that for all $i \in I_{n}, \tilde{k}_{i}$ and $\hat{k}_{i}$ are only dependent on the constant biological parameters of the model. Moreover, if the conditions (58) are satisfied, then $\bar{N}_{i}>0$, for all $i \in I_{n}$.

Proof: For the sake of brevity, we give only the outlines of the proof. First, using (55), we check that the derivative of the function $Q\left(\tilde{x}_{1}(t)\right)$, introduced in (32), along the trajectories of the nonlinear system (31) satisfies

$$
\begin{aligned}
\dot{Q}(t) \leq & 2\left[-\beta_{1 *}+L_{1}\left|\mu_{1}\right| C_{1}\right] Q\left(\hat{x}_{1}(t)\right) \\
& +2 L_{1}\left(\left|\mu_{1}\right|+\hat{s}_{1}\left|\hat{x}_{1}(t)\right|\right) I_{1}\left(\hat{x}_{1 t}\right) \\
& +\hat{s}_{1}\left|\hat{x}_{1}(t)\right| Q\left(\hat{x}_{1}(t)\right) .
\end{aligned}
$$

Then, using (60) in conjunction with the fact that $\left|\hat{x}_{1}(t)\right| \leq$ $2 \sqrt{N_{1}\left(\hat{x}_{1 t}\right)}$, we determine the following upper bound for the derivative of the functional $N_{1}$, introduced in (39), along the trajectories of the nonlinear system (31),

$$
\begin{aligned}
& \left.\dot{N}_{1}(t) \leq-\tilde{\varsigma}_{1} N_{1}\left(\hat{x}_{1 t}\right)+\left[\hat{s}_{1} \sqrt{N_{1}\left(\hat{x}_{1 t}\right.}\right)-\frac{\varsigma_{1}}{4}\right] Q_{1}\left(\hat{x}_{1}(t)\right) \\
& +\left[2 L_{1} \hat{s}_{1} \sqrt{N_{1}\left(\hat{x}_{1 t}\right)}-\frac{\varsigma_{1} e^{-\tau_{1}}}{2 C_{1}}\right] I_{1}\left(\hat{x}_{1 t}\right)-\frac{\varsigma_{1}}{8} Q\left(\hat{x}_{1}(t)\right) .
\end{aligned}
$$

Now, observe that if the condition (59) is satisfied then

$$
\dot{N}_{1}(t) \leq-\tilde{\varsigma}_{1} N_{1}\left(\hat{x}_{1 t}\right)-\frac{\varsigma_{1}}{8} Q\left(\hat{x}_{1}(t)\right) .
$$

This allows us to conclude that the origin of the system (31) is exponentially stable with a decay rate smaller than $\tilde{\varsigma}_{1}$ when $i=1$, for all initial conditions satisfying (59). This result is generalized to the overall system, $i \in I_{n}$, by similar arguments as in Theorem 2.

\section{CONCLUSIONS}

We proposed explicit constructions of suitable strict Lyapunov functionals for nonlinear systems with distributed delays. In contrast with the methods already used, the Lyapunov approach allowed us to address more general statements, as the case of time-varying parameters and the approximation of the basin of attraction of the positive steady state. Furthermore, compared to the previous works where the cell division probability density function (mitosis) was taken as a Dirac mass function in [1], and assumed to be a truncated exponential term in [10], our results were derived without any extra assumption on the mitosis function. For lack of space, we have not been able to illustrate some simulations that validate our theoretical results. Finally, it is worth mentioning that we are working on the identification of the parameters involved in the mathematical models.

\section{REFERENCES}

[1] M. Adimy, F. Crauste, A. Abdllaoui, Discrete Maturity-Structured Model of Cells Differentiation with Applications to Acute Myelogenous Leukemia, J. Biological Systems, No. 3, pp. 395-424, 2008.

[2] J. L. Avila, C. Bonnet, J. Clairambault, H. Özbay, S. Niculescu, F. Merhi, A. Ballesta, R. Tang, and J.-P. Marie. Analysis of a new model of cell population dynamics in acute myeloid leukemia. In Advances in Delay and Dynamics, pp. 315-328. Springer, 2014.

[3] W. Djema, F. Mazenc, C. Bonnet, Lyapunov Stability Analysis of a Model Describing Hematopoiesis. European Control Conference, Linz, Austria, pp. 2711-2716, 2015.

[4] E. Fridman, C. Bonnet, F. Mazenc, W. Djema, Stability of the cell dynamics in Acute Myeloid Leukemia. Systems \& Control Letters, 2016.

[5] V. Kolmanovskii, A. Myshkis, Introduction to the Theory and Applications of Functional Differential Equations, Kluwer Academic Publishers, Dordrecht, 1999.

[6] M.C. Mackey, Unified hypothesis of the origin of plastic anemia and periodic hematopoiesis, Blood, 51: pp. 941-956, 1978.

[7] M. Malisoff, F. Mazenc, Constructions of Strict Lyapunov Functions, Serie : Communications and Control Engineering. Spinger-Verlag London Ltd, U.K., 2009.

[8] F. Mazenc, S.-I. Niculescu, M. Krstic, Lyapunov-Krasovskii Functionals and Application to Input Delay Compensation for Linear TimeInvariant Systems. Automatica, Vol. 48, Issue 7, pp. 1317-1323, July 2012.

[9] J.D. Murray, Mathematical Biology, 18, Berlin: Springer, 2nd Edition, 1993.

[10] H. Özbay, C. Bonnet, H. Benjelloun, J. Clairambault, Stability analysis of cell dynamics in leukemia. Math. Model Nat. Phenom., Vol. 7, No. 1, pp. 203-234, 2012.

[11] L. Pujo-Menjouet, S. Bernard, M.C. Mackey, Long period oscillations in a $G_{0}$ model of hematopoietic stem cells, SIAM. J. Appl. Dynam. Syst. 4(2): pp. 312-332, 2005.

[12] M. Vidyasagar, Nonlinear Systems Analysis, Second Edition, Classics in Applied Mathematics, Siam, 2002. Berlin: Springer, 2nd Edition, 1993. 